\title{
Instanton vibrations of the 3-Skyrmion
}

\author{
Conor J. Houghton* \\ Department of Applied Mathematics and Theoretical Physics, University of Cambridge, Silver Street, \\ Cambridge CB3 9EW, United Kingdom \\ (Received 27 May 1999; published 1 October 1999)
}

\begin{abstract}
The Atiyah-Drinfeld-Hitchin-Manin matrix corresponding to a tetrahedrally symmetric 3-instanton is calculated. Some small variations of the matrix correspond to vibrations of the instanton-generated 3-Skyrmion. These vibrations are decomposed under tetrahedral symmetry and this decomposition is compared to previous knowledge of the 3-Skyrmion vibration spectrum. [S0556-2821(99)05420-X]
\end{abstract}

PACS number(s): 12.39.Dc, 11.27.+d

\section{INTRODUCTION}

In the Skyrme model, the classical $B$-nucleon nucleus is a $B$-Skyrmion: a minimum energy Skyrme field with topological charge $B$. The $B$-Skyrmions have been calculated numerically for $B$ up to nine $[1,2]$.

The Skyrme model is nonrenormalizable and so cannot be quantized as a field theory. However, it is hoped that the quantum mechanics on some finite-dimensional space in the charge $B$ sector might give a good model of the quantized $B$-nucleon. This approach has been reasonably successful in the 1-Skyrmion case [3] but for higher $B$ it is hard to choose a suitable, tractable, finite-dimensional space.

The 1-Skyrmion is spherically symmetric and has six zero modes: three translational and three rotational. This suggests that the finite-dimensional space should be $6 \mathrm{~B}$-dimensional and one popular candidate is the gradient-flow manifold descending from the charge $B$ spherical saddle-point [4]. Recently, the vibration spectra of $B$-Skyrmions have been calculated numerically for $B$ equals two, three, four and seven $[5,6]$. It was found that the vibration frequencies of the $B$-Skyrmion are divided into two groups by the breather mode which corresponds to dilation. This suggests that it might be necessary to add the breather mode to the $6 B$-dimensional space to give $(6 B+1)$-dimensions, or even to include seven dimensions for each Skyrmion to give a $7 B$-dimensional space. Another suggestion is that $8 B-3$ vibrational modes should be expected [7].

The modes below the breather have been interpreted as being monopole-like and may correspond to the gradientflow manifold descending from the saddle-points of infinite Skyrmion separation and from the charge $B$ torus [8]. It may be that this is the space upon which the quantization should be performed. It is a $(4 B+2)$-dimensional space.

All these spaces are thought to be well approximated by instanton generated Skyrme fields $[9,10]$. In the instanton construction, Skyrme fields are derived from instanton fields by calculating their holonomy in the $x_{4}$ direction [9]. There is a $(8 B-1)$-dimensional family of baryon number $B$ Skyrme fields derived from the space of $B$-instantons. It is known for $B$ equals one, two, three and four that the $B$-Skyrmion is well approximated by an instanton-generated

*Email address: C.J.Houghton@damtp.cam.ac.uk
Skyrme field $[9,11]$. In this paper the vibrations around the instanton generated 3-Skyrmion are studied. The decomposition of these vibrations as representations of the tetrahedral group includes the same representations as are found in the decomposition of the numerically determined spectrum. This seems to indicate that the numerically determined vibrations are close to being tangent to the space of instanton generated Skyrme fields. It is consistient with the view that, whatever space should be used to quantize the $B$-Skyrmion, it is approximated by a subspace of the space of instanton generated Skyrme fields.

The 3-Skyrmion has tetrahedral symmetry [1]. In [11], the Jackiw-Nohl-Rebbi (JNR) ansatz [12] is used to derive a tetrahedral 3-instanton. From this, the instanton-generated 3-Skyrmion is calculated. In [13], Walet examines vibration modes of the instanton-generated 3-Skyrmion by varying the JNR parameters. Although a large class of instantons can be constructed using the JNR ansatz, it is not general. However, the Atiyah-Drinfeld-Hitchin-Manin (ADHM) construction [14-16] is general and, in this paper, the tetrahedral 3-instanton ADHM matrix is calculated. The instantongenerated 3-Skyrmion vibration modes are then examined by varying the ADHM parameters. The vibration frequencies are not calculated. However, the vibrations are decomposed under the action of the tetrahedral symmetry. This allows the decomposition to be compared to other calculations of 3-Skyrmion vibrations.

\section{THE ADHM MATRIX FOR THE 3-SKYRMION}

Symmetric ADHM matrices have been discussed in a recent paper by Singer and Sutcliffe [17] and this should be consulted for any details not included in this section.

The ADHM matrix for a $B$-instanton is a quaternionic matrix

$$
\hat{M}=\left(\begin{array}{c}
L \\
M
\end{array}\right)
$$

where $L$ is a $B$-vector and $M$ is a symmetric $B \times B$ matrix. $\hat{M}$ must satisfy the ADHM constraint 
Dagger denotes quaternionic conjugation and matrix transposition.

Pure quaternions can be identified with $\mathrm{SU}_{2}$ by $-i \boldsymbol{\sigma}$ $=(i, j, k)$ where $\boldsymbol{\sigma}=\left(\sigma_{1}, \sigma_{2}, \sigma_{3}\right)$ are the Pauli matrices. With this identification, the instanton gauge fields are

$$
A_{\mu}(x)=N^{\dagger}(x) \partial_{\mu} N(x)
$$

where $N(x)$ is the unit length $(B+1)$-vector solving

$$
N^{\dagger}(x)\left(\begin{array}{c}
L \\
M-x \mathbf{1}_{B}
\end{array}\right)=0 .
$$

In this equation, $\mathbf{1}_{B}$ is the $B \times B$ identity matrix and the $\mathbf{R}^{4}$ position is written as a quaternion: $x=x_{4}+x_{1} i+x_{2} j+x_{3} k$.

There is an ambiguity in choosing $N(x)$ given by

$$
N(x) \rightarrow N(x) g(x)
$$

where $g(x)$ is a unit quaternion. The unit quaternions are identified with the two-dimensional representation of $\mathrm{SU}_{2}$ and so this ambiguity corresponds to gauge transformations of the fields. There is also an ambiguity in $\hat{M}$ given by

$$
\hat{M} \rightarrow\left(\begin{array}{cc}
g & 0 \\
0 & \rho
\end{array}\right) \hat{M} \rho^{-1}
$$

where $g$ is a unit quaternion and $\rho$ is a real orthogonal $B$ $\times B$ matrix. This is a gauge transformation of the ADHM matrix: it does not affect the fields.

This convenient version of the ADHM data is the canonical form discussed in $[15,16]$. The ADHM construction, as originally introduced, involved a larger gauge ambiguity and a second ADHM matrix: a matrix coefficient of $x$ in (4). The canonical form is a partial fixing of the larger gauge ambiguity.

Under the conjugate action of unit quaternions on $x$, the real part of $x$ is fixed and the imaginary part transforms under the three-dimensional representation of $\mathrm{SO}_{3}$. This means that for a spatial rotation $R$ there is a quaternion $g$ so that

$$
x_{4}+(i j k) R\left(\begin{array}{c}
x_{1} \\
x_{2} \\
x_{3}
\end{array}\right)=g x g^{-1} \text {. }
$$

Of course, $-g$ corresponds to the same $R: \mathrm{SU}_{2}$ is a double cover of $\mathrm{SO}_{3}$.

As explained in [17], an instanton has the spatial rotation symmetry $x \rightarrow g x g^{-1}$ for unit quaternion $g$ if

$$
\left(\begin{array}{c}
L \\
M-g x g^{-1} \mathbf{1}_{B}
\end{array}\right)=\left(\begin{array}{cc}
\tilde{g} & 0 \\
0 & \rho \cdot g
\end{array}\right)\left(\begin{array}{c}
L \\
M-x \mathbf{1}_{B}
\end{array}\right) g^{-1} \cdot \rho^{-1}
$$

where $\tilde{g}$ is a unit quaternion and $\rho \cdot g$ is the product of the real orthogonal matrix $\rho$ and the unit quaternion $g$. Thus, the ADHM matrix is symmetric if the spatial rotation is equivalent to a gauge transformation. If the instanton is symmetric under some subgroup of $\mathrm{SO}_{3}$ then the collection of $\rho$ 's and of $\tilde{g}$ 's form a real $B$-dimensional representation and a complex two-dimensional representation of the corresponding binary subgroup of $\mathrm{SU}_{2}$.

\section{A. The tetrahedrally symmetric ADHM matrix}

Since the 3-Skyrmion is tetrahedrally symmetric, the corresponding ADHM matrix is also tetrahedrally symmetric. The tetrahedral group $T$ is the twelve element subgroup of $\mathrm{SO}_{3}$ which, in one orientation, is generated by a rotation of $\pi$ about the $x_{3}$-axis and a rotation of $2 \pi / 3$ about $x_{1}=x_{2}$ $=x_{3}$. These generators will be called $r$ and $t$ respectively and the corresponding unit quaternions are $g(r)=k$ and $g(t)$ $=(1-i-j-k) / 2$.

The group is isomorphic to the alternating group $\mathfrak{A}_{4}$. The tetrahedral double group is the 24 element subgroup of $\mathrm{SU}_{2}$ which double covers the tetrahedral group. The representation theory of the tetrahedral group is described in, for example, Hamermesh [18]. There are one, two and three dimensional representations derived by restricting the one, two and three dimensional irreducible representations of $\mathrm{SU}_{2}$ to the tetrahedral group. They are $A=\left.1\right|_{T}, E^{\prime}=\left.2\right|_{T}$ and $F$ $=\left.3\right|_{T}$ where $n$ denotes the irreducible $n$-dimensional representation of $\overline{\mathrm{SU}}_{2}$. There is, in addition, the two-dimensional representation $E$ and the four-dimensional representation $G^{\prime}$. These representations are reducible into conjugate pairs of representations with complex characters.

The ADHM matrix

$$
\hat{M}_{T}=\left(\begin{array}{c}
L_{T} \\
M_{T}
\end{array}\right)=\left(\begin{array}{ccc}
i & j & k \\
0 & k & j \\
k & 0 & i \\
j & i & 0
\end{array}\right)
$$

is tetrahedrally symmetric. This matrix was found by trial and error. Having written down a likely form of the matrix it is easy to check whether or not it has the required symmetries. Explicitly, the matrices giving the compensating gauge transformations are

$$
\rho(r)=\left(\begin{array}{ccc}
-1 & 0 & 0 \\
0 & -1 & 0 \\
0 & 0 & 1
\end{array}\right)
$$

with $\tilde{g}(r)=g(r)$ for $r$ and

$$
\rho(t)=\left(\begin{array}{lll}
0 & 1 & 0 \\
0 & 0 & 1 \\
1 & 0 & 0
\end{array}\right)
$$

with $\tilde{g}(t)=g(t)$ for $t$. Thus, in this case the $\rho$ 's form the representation $F$ and the $\tilde{g}$ 's form the representation $E^{\prime}$.

$\hat{M}_{T}$ is not just symmetric under $T$. It is also symmetric under the 24 element group $T_{d}$ which extends $T$ by the $S_{4}$ element $u$ :

$$
u:\left(x_{1}, x_{2}, x_{3}, x_{4}\right) \rightarrow\left(-x_{2}, x_{1},-x_{3},-x_{4}\right) .
$$


In fact,

$$
\left(\begin{array}{c}
L \\
M-u(x) \mathbf{1}_{B}
\end{array}\right)=-\left(\begin{array}{cc}
-1 & \\
& \rho
\end{array}\right) \frac{1-k}{\sqrt{2}}\left(\begin{array}{c}
L \\
M-x \mathbf{1}_{3}
\end{array}\right) \frac{1+k}{\sqrt{2}} \rho^{-1}
$$

where

$$
\rho=\left(\begin{array}{ccc}
0 & 1 & 0 \\
-1 & 0 & 0 \\
0 & 0 & 1
\end{array}\right)
$$

The representation theory for $T_{d}$ is also described in Hamermesh [18]. The vector representation $F$ of $T$ is replaced by a true vector $F_{2}$ and an axial vector $F_{1}$. In the same way, the trivial representation $A$ is replaced by a true scalar $A_{1}$ and a pseudo-scalar $A_{2}$. Although reducible as a representation of $T, E$ is irreducible as a representation of $T_{d}$. There are similar changes to the double group representations.

\section{B. Uniqueness and the tetrahedrally symmetric ADHM matrix}

$\hat{M}_{T}$ is not unique, there is a two-parameter family of tetrahedral matrices given by $x\left(\hat{M}_{T}+y \mathbf{1}_{4}\right)$. $y$ can be set to zero. It corresponds to translation of the instanton in the $x_{4}$ direction and this does not change the corresponding Skyrme field. $x$ is a scale parameter and, when calculating the instanton-generated Skyrmion, the scale is fixed by minimization of the Skyrme energy. There can be no more than two parameters because, as explained in [17], it follows from (8) that

$$
M_{T}:\left.\left.\underline{3}\right|_{T} \rightarrow(\underline{3} \times \underline{2} \times \underline{2})\right|_{T}
$$

that is

$$
M_{T}: F \rightarrow 3 F+E+A
$$

and so there is a three-parameter family of candidate $M_{T}$ matrices. This is exhausted by

$$
\left(\begin{array}{ccc}
d & q k & r j \\
r k & d & q i \\
q j & r i & d
\end{array}\right) .
$$

Similarly,

$$
L_{T}:\left.\left.(\underline{3} \times \underline{2})\right|_{T} \longrightarrow \underline{2}\right|_{T}=E^{\prime} .
$$

and, since $\left.(\underline{3} \times \underline{2})\right|_{T}=E^{\prime}+G^{\prime}$, there is a one-parameter family of $L_{T}$. The symmetry of $M_{T}$ and the ADHM constraint (2) reduce these four parameters to the two parameters $x$ and $y$ above. There is another two-parameter family of symmetric matrices corresponding to the dual tetrahedron. This is given by replacing $M_{T}$ in $\hat{M}_{T}$ by $-M_{T}$.

It is possible to translate between JNR data and ADHM matrices. This is useful here because it gives an explicit verification that the 3-Skyrmion generating instanton of [11] lies in the one-parameter family $x \hat{M}_{T}$. The general formula, translating JNR data into ADHM data, is given in Section 5 of [15]. Unfortunately, these ADHM data are not in the canonical form involving a single ADHM matrix. It seems that it is difficult to write the general JNR-derived ADHM data in canonical form. However, in the particular case of interest here, a straight forward calculation shows that $\hat{M}_{T}$ is the canonical form of ADHM data derived from tetrahedral JNR data.

\section{VARIATIONS}

Small variations around $\hat{M}_{T}$ are now considered. Writing

$$
\hat{M}=\hat{M}_{T}+\hat{m}
$$

$\hat{m}$ satisfies the linearized ADHM constraint

$$
\operatorname{Im}\left(\hat{m}^{\dagger} \hat{M}_{T}+\hat{M}_{T}^{\dagger} \hat{m}\right)=0
$$

If

$$
\hat{m}=\left(\begin{array}{ccc}
l_{1} & l_{2} & l_{3} \\
m_{11} & m_{12} & m_{13} \\
m_{12} & m_{22} & m_{23} \\
m_{13} & m_{23} & m_{33}
\end{array}\right)
$$

the linearized equations are

$$
\begin{aligned}
& \operatorname{Im}\left(\bar{l}_{1} j+\bar{m}_{11} k+\bar{m}_{13} i-i l_{2}-k m_{22}-j m_{23}\right)=0, \\
& \operatorname{Im}\left(\bar{l}_{1} k+\bar{m}_{11} j+\bar{m}_{12} i-i l_{3}-k m_{23}-j m_{33}\right)=0, \\
& \operatorname{Im}\left(\bar{l}_{2} k+\bar{m}_{22} i+\bar{m}_{12} j-j l_{3}-k m_{13}-i m_{33}\right)=0,
\end{aligned}
$$

where bar denotes quaternionic conjugation. These equations can be solved to give expressions for nine of the twelve $l_{i}$ parameters. The remaining three components correspond to the gauge transformation

$$
\hat{M}_{T} \rightarrow\left(\begin{array}{cc}
g & 0 \\
0 & \mathbf{1}_{3}
\end{array}\right) \hat{M}_{T}
$$

This gauge freedom can be fixed by requiring, for example, that $l_{1}$ is proportional to $i$. In this way, $l$ may be completely determined by $m$ and by gauge fixing.

In order to decompose the $24 m_{i j}$ components as representations of $T_{d}$, the actions of $r, t$ and $u$ on $\hat{M}_{T}$ are considered. Thus, for example,

$$
\begin{aligned}
\rho(r) g(r) m g(r)^{-1} \rho(r)^{-1} & \\
= & \left(\begin{array}{ccc}
-k m_{11} k & -k m_{12} k & k m_{13} k \\
-k m_{12} k & -k m_{22} k & k m_{23} k \\
k m_{13} k & k m_{23} k & -k m_{33} k
\end{array}\right)
\end{aligned}
$$

and so the character of $r$ is zero. The characters of $t$ and $u$ can be calculated in the same way: they are also zero. This means that the decomposition of the $m_{i j}$ is 


$$
A_{1}+A_{2}+2 E+3 F_{1}+3 F_{2} \text {. }
$$

Not all of these multiplets correspond to Skyrmion vibrations. There remains the gauge freedom

$$
M_{T} \rightarrow \rho M_{T} \rho^{-1} \text {. }
$$

By considering an infinitesimal $\rho$ and calculating the character, it is found that this variation is an $F_{1}$. The remaining 21 variations correspond to the 21 dimensions of the space of 3 -instantons. However, the variation corresponding to time translation, $A_{2}$, does not affect the 3-Skyrmion and is discarded. Twenty variational modes remain: six of these correspond to zero modes. In fact, the translation and rotation zero modes of the Skyrmion correspond to an $F_{2}$ and an $F_{1}$ respectively. Thus, the instanton modes of the 3-Skyrmion decompose as

$$
A_{1}+2 E+2 F_{1}+3 F_{2}
$$

under $T_{d}$ and, of these, one $F_{1}$ and one $F_{2}$ are zero modes and the rest are vibrational modes. The isospin zero modes are not included in this decomposition because they do not correspond to variations of the ADHM matrix.

\section{DISCUSSION}

The $A_{1}$ is the breather mode corresponding to dilation. In the numerical results of Baskerville, Barnes, and Turok [5], it appears in the middle of the vibration spectrum. In order of increasing frequency and ignoring radiation, Baskerville, Barnes, and Turok find the spectrum to be

$$
F_{2}+E+A_{1}+F_{2}+E \text {. }
$$

The $E+F_{2}$ below the breather are the modes described in [8] as monopole modes. They correspond to variations of the rational map parameters in the rational map ansatz of [8].

The $E+F_{2}$ above the breather are discussed in [7] by Baskerville and Michaels. It has been observed that, to a good approximation, there are $2 B-2$ straight lines of zero baryon density, known as branch lines, radiating from the center of a $B$-Skyrmion. In [7], the variations of the angular positions of the branch lines are parametrized. These are then decomposed. It is noted that if an axial vector is removed from this decomposition, the decomposition then matches the super-breather modes in the 2-Skyrmion and 3-Skyrmion spectra. In the 4-Skyrmion case the decomposition is consistent with the observed spectrum. Baskerville and Michaels interpret the axial vector which must be removed as the axial vector of rotational zero modes.

Some of the monopole modes also change the branch line positions. Therefore, [7] implies that the super-breather mode decomposition duplicates part of the decomposition of the monopole modes. There are, in total, $4 B+2$ monopole modes of a $B$-Skyrmion. Six of them do not change the branch lines: of these, three are the isospin zero modes. Because the parameters in the rational map are complex, the monopole modes come in pairs of opposite parity. There are three monopole modes which compose pairs with isospin modes in this way. These are the other three modes which do not change the positions of branch lines. The remaining $4 B$ -4 modes change the positions of the branch lines. These $4 B-4$ modes include the three translational and three rotational zero modes along with $4 B-10$ vibrational modes. Thus, there are $4 B-7$ monopole modes which are not rotational zero modes and which change the positions of the branch lines. It is possible to reformulate the observation made in [7]: the decomposition of these $4 B-7$ modes duplicates the decomposition of the super-breather modes.

Thus, an exception is made for the rotational zero modes, the translational zero modes are duplicated but the rotational zero modes are not. In fact, in the $B=3$ case, the instanton modes contain a $F_{1}$ duplicating the rotational zero modes. This $F_{1}$ has not been observed numerically. The reason for this may be that the $F_{1}$ mode has a rather high frequency.

For $B=3$, the monopole modes which fix the branch line positions are an $F_{1}$ of isospin and an $F_{2}$ vibration. The monopole modes which change the branch line positions are the rotational and translational zero modes $F_{1}+F_{2}$ and the multiplet of vibration modes $E$. Thus, the $E$ in the superbreather part of the 3-Skyrmion spectrum duplicates the $E$ in the monopole part. The $F_{2}$ duplicates the translational zero modes. If a duplicate is also included for the rotational zero modes, then the aggregate of the breather, the monopole modes and their duplicates match the instanton mode decomposition (27).

Because Walet uses JNR ansatz instantons, not all of the instanton modes are included in the harmonic analysis of [13]. To be precise, there is only one $E$, whereas the ADHM construction gives two. In the case of the rational map decomposition, it is known that the $E$ is spanned by tangent vectors lying along the $S_{4}$ symmetric geodesics. These are referred to in [19] as twisted line scattering geodesics.

There is a three-dimensional family of ADHM matrices, symmetric under the $D_{2 d}$ generated by $u$ and $\pi$ rotations about the Cartesian axes. Vibrations tangent to this family lie in the $A_{1}+2 E$ of the decomposition (27). The symmetric ADHM matrices are

$$
\hat{M}_{D_{2 d}}=\left(\begin{array}{ccc}
a i & a j & b k \\
e & c k & d j \\
c k & -e & d i \\
d j & d i & 0
\end{array}\right)
$$

with, from the ADHM constraint,

$$
\begin{aligned}
& a b+d e-d c=0 \\
& d^{2}-a^{2}+2 e c=0 .
\end{aligned}
$$

For $a=b=c=d=x$ and $e=0$ this is $\hat{M}_{T}$ and for $a=c=d$ $=e=0$ it is axially symmetric about the $x_{3}$-axis. Translating $D_{2 d}$ JNR data to ADHM data and rewriting it in canonical form gives the two-parameter subfamily with $a=d, c=b$ and $e=0$. However, as noted by Walet, this subfamily of $D_{2 d}$ symmetric ADHM matrices shares the curious feature of the $T_{h}$ matrices in [17], it does not include well-separated instantons of equal scale. More complicated subfamilies, in- 
cluding well-separated instantons of equal scale, may be chosen by using arguments similar to those in [17]. One simple example, with $b=1$ fixing the scale, is

$$
e=\frac{c\left(c^{2}-1\right)}{2\left(c^{4}+1\right)} .
$$

Of course, this is just one path which passes though the various features associated with twisted line scattering. The infinitesimal behaviur around $\hat{M}_{T}$ does not give the splitting of the $2 E$ into a sub-breather $E$ and the super-breather $E$. It is not known how to make this split, without calculating the holonomy and performing the full harmonic analysis as Walet did for JNR ansatz instanton-generated 3-Skyrmions.
In conclusion, the instanton modes of the 3-Skyrmion have been calculated and decomposed. The decomposition fits well with other similar decompositions. The primary question provoked by the calculations is whether it is possible to split the modes further without undertaking the harmonic analysis.

\section{ACKNOWLEDGMENTS}

The financial assistance of Fitzwilliam College, Cambridge is gratefully acknowledged. This work is supported, in part, by PPARC. I am grateful to Kim Baskerville for useful discussion.
[1] E. Braaten, S. Townsend, and L. Carson, Phys. Lett. B 235, 147 (1990).

[2] R.A. Battye and P.M. Sutcliffe, Phys. Rev. Lett. 79, 363 (1997).

[3] G. Atkins, C.R. Nappi, and E. Witten, Nucl. Phys. B228, 552 (1983).

[4] N.S. Manton, Phys. Rev. Lett. 60, 1916 (1988).

[5] W.K. Baskerville, C. Barnes, and N.G. Turok, talk given at Solitons conference: Kingston, Ontario, 1997.

[6] W.K. Baskerville, Vibrational spectrum of the $B=7$ Skyrme soliton, hep-th/9906063.

[7] W.K. Baskerville and R. Michaels, Phys. Lett. B 488, 275 (1999).

[8] C.J. Houghton, N.S. Manton, and P.M. Sutcliffe, Nucl. Phys. B510, 507 (1998).

[9] M.F. Atiyah and N.S. Manton, Phys. Lett. B 222, 438 (1989).
[10] M.F. Atiyah and N.S. Manton, Commun. Math. Phys. 152, 391 (1993).

[11] R.A. Leese and N.S. Manton, Nucl. Phys. A572, 575 (1994).

[12] R. Jackiw, C. Nohl, and C. Rebbi, Phys. Rev. D 15, 1642 (1977).

[13] N.R. Walet, Nucl. Phys. A606, 429 (1996).

[14] M.F. Atiyah, N.J. Hitchin, V.G. Drinfeld, and Yu.I. Manin, Phys. Lett. 65A, 185 (1978).

[15] E.F. Corrigan, D.B. Fairlie, S. Templeton, and P. Goddard, Nucl. Phys. B140, 31 (1978).

[16] N.H. Christ, E.J. Weinberg, and N.K. Stanton, Phys. Rev. D 18, 2013 (1978).

[17] M.A. Singer and P.M. Sutcliffe, Nonlinearity 12, 987 (1999).

[18] M. Hamermesh, Group Theory and its Applications to Physical Problems (Addison-Wesley, Reading, 1962).

[19] C.J. Houghton and P.M. Sutcliffe, Nucl. Phys. B464, 59 (1996). 